Published in final edited form as:

Nat Photonics. 2014 July ; 8(7): 550-555. doi:10.1038/nphoton.2014.114.

\title{
Emitters of $\mathrm{N}$-photon bundles
}

\author{
C. Sánchez Muñoz ${ }^{1}$, E. del Valle ${ }^{1}$, A. González Tudela ${ }^{2}$, K. Müller ${ }^{3}$, S. Lichtmannecker ${ }^{3}$, M. \\ Kaniber $^{3}$, C. Tejedor ${ }^{1}$, J.J. Finley ${ }^{3}$, and F.P. Laussy ${ }^{1}$ \\ ${ }^{1}$ Condensed Matter Physics Center (IFIMAC), Departamento de Física Teórica de la Materia \\ Condensada, Universidad Autónoma de Madrid, 28049 Madrid, Spain \\ ${ }^{2}$ Max-Planck-Institut für Quantenoptik, Hans-Kopfermann-Str. 1, 85748 Garching, Germany \\ ${ }^{3}$ Walter Schottky Institut, Technische Universï̈at München, Am Coulombwall 4, 85748 Garching, \\ Germany
}

\section{Abstract}

Controlling the ouput of a light emitter is one of the basic tasks of photonics, with landmarks such as the laser and single-photon sources. The development of quantum applications makes it increasingly important to diversify the available quantum sources. Here, we propose a cavity QED scheme to realize emitters that release their energy in groups, or "bundles" of $N$ photons, for integer $N$. Close to $100 \%$ of two-photon emission and $90 \%$ of three-photon emission is shown to be within reach of state of the art samples. The emission can be tuned with system parameters so that the device behaves as a laser or as a $N$-photon gun. The theoretical formalism to characterize such emitters is developed, with the bundle statistics arising as an extension of the fundamental correlation functions of quantum optics. These emitters will be useful for quantum information processing and for medical applications.

\begin{abstract}
The photon is the building block of light. Every state of the electromagnetic field comes as a superposition of photons, even classical states, which are Poisson distributions. Particular combinations of photons-from more stringent distributions to entangled superpositionsare required to power quantum technology. A privileged platform to sculpt desirable states of light is Cavity Quantum Electrodynamics (cQED) ${ }^{1}$. These laboratories of the extreme allow the control of the interaction of light with matter at the ultimate quantum limit. We show here how they can be used to realize a family of $N$-photon emitters, i.e., sources that release their energy exclusively in groups, or bundles, of $N$ photons (for integer $N$ ) and in effect provide us with light made up from building blocks that are not single photons anymore. This ability to substitute the quantum of light by a bundle has unforeseeable consequences for both applications and fundamental physics. For instance, this renormalizes
\end{abstract}

\footnotetext{
Users may view, print, copy, and download text and data-mine the content in such documents, for the purposes of academic research, subject always to the full Conditions of use:http://www.nature.com/authors/editorial_policies/license.html\#terms

Correspondence and requests for materials should be addressed to FPL (fabrice.laussy@ gmail.com). Author Contributions: FPL and EdV proposed the idea. CSM, EdV and FPL developped the theoretical formalism and the conceptual tools. CSM implemented the theoretical methods and analyzed the data. AGT, KM, SL, MK and JJF contributed material, analysis tools and expertise. FPL, CSM, EdV, CT and JJF wrote the main paper. CSM, EdV, AGT and FPL wrote the supplementary information. FPL supervised the research. All the authors discussed the results and its implications and commented on the manuscript. PACS numbers: 42.50.Ct, 42.50.Ar, 42.72.-g, 02.70.Ss
} 
the link between the energy of the fundamental unit of excitation to its frequency through a magnified Planck constant: $E=N h v$. The type of emission can be varied with system parameters to realize both $N$-photon lasers and photon guns ${ }^{2}$ at the $N$-photon level. Such highly non-classical emitters should boost new generations of light sources ${ }^{3,4}$, be useful to produce NOON states ${ }^{5}$, for quantum lithography and metrology ${ }^{6}$, and also for medical applications, allowing for higher penetration lengths and increased resolution with minimum harm to the tissues ${ }^{7,8}$. The recent demonstration that biological photoreceptors are sensitive to photon statistics ${ }^{9}$ may also render such sources highly relevant for studies of biological photosystems and, potentially, of quantum biology ${ }^{10}$.

\section{Transition from Jaynes-Cummings to Mollow dynamics}

Our scheme relies on the paradigm of cQED: one two-level system in a cavity (Fig. 1). This is realized in a wealth of physical systems, ranging from atoms in optical cavities ${ }^{11}$ to superconducting qubits in microwave resonators ${ }^{12}$ and quantum dots in microcavities ${ }^{13}$. The dynamics is described by the Jaynes-Cummings Hamiltonian $H_{0}=\omega_{a} a^{\dagger} a+\omega_{\sigma} \sigma^{\dagger} \sigma+g\left(a^{\dagger} \sigma\right.$ $+\sigma^{\dagger} a$ ) with $a$ and $\sigma$ the second quantization lowering operators of the light field (boson statistics) and the Quantum Emitter (QE, two-level system), respectively, with corresponding free energies $\omega_{a}$ and $\omega_{\sigma}$ and coupling strength $g^{14}$. The configuration under study is the resonant excitation by an external laser of the $\mathrm{QE}^{15-17}$ far in the dispersive regime with the cavity $\left(\left|\omega_{a}-\omega_{\sigma}\right| \gg g \sqrt{N+1}\right){ }^{18-20}$. The energy structure of $H_{0}$ is shown in Fig. 2a with the QE at $\Delta g=-60\left(\Delta=\omega_{a}-\omega_{\sigma}\right)$, in which case the states are essentially the bare ones. The laser of frequency $\omega_{\mathrm{L}}$ and pumping intensity $\Omega$ is included by adding $\Omega\left(e^{-i \omega L t} \sigma^{\dagger}+e^{i \omega \mathrm{L} t} \sigma\right)$ to $H_{0}$. We assume the rotating wave approximation, whose validity is justified in the Supplementary Information. At pumping low enough not to distort the level structure, one can excite selectively a state with $N$ photon(s) in the cavity at the $(N+1)$ th rung by adjusting the laser frequency to

$$
\omega_{N} \approx \omega_{a}+\frac{\sqrt{4(N+1) g^{2}+\Delta^{2}}-\Delta}{2(N+1)},
$$

with $N \in \mathrm{N}^{21}$. This is shown in Fig. 2a for the case $N=2$, corresponding to the excitation of the third rung, with a photon-blockade ${ }^{22,23}$ at all other rungs (above and below) ${ }^{24,25}$. The positions of the resonances are shown in Fig. 3b. In the absence of dissipation, exciting a resonance leads to the generation of an exotic brand of maximally entangled polaritons, of the type $(|0 g\rangle \pm|N e\rangle) / \sqrt{2}$ rather than the usual case $(|0 e\rangle+|1 g\rangle) / \sqrt{2}$. The dynamics of the system for the case $N=2$ is presented in Fig. 4a. Strikingly, full amplitude Rabi oscillations between the $\mid 0 \mathrm{~g} /$ and $|N \mathrm{e}\rangle$ states are observed. Further characterization of these remarkable quantum states is provided in the Supplementary Information. We proceed towards the configuration that will bring such resonances to fruition in terms of applications.

When increasing pumping, resonances in the amplitude of the Rabi oscillations persist but are blueshifted due to the dressing of the states by the laser. The level structure becomes that of a dressed atom ${ }^{26}$ strongly detuned from a cavity mode ${ }^{27}$, bridging the Jaynes-Cummings dynamics with another fundamental model of light-matter interaction, namely, the Mollow 
physics of resonance fluorescence ${ }^{28}$. The strong coupling that was previously dominated by the interaction between the $\mathrm{QE}$ and a cavity-photon, and probed by the laser, is now dominated by the interaction of the $\mathrm{QE}$ with the laser-photons, and is probed by the cavity. This elegant transition between the two pillars of nonlinear quantum optics brings the resonances in Eq. (1) to the corresponding form:

$$
\omega_{N}(\Omega) \approx \omega_{a}+\frac{\sqrt{4\left(N^{2}-1\right) \Omega^{2}+N^{2} \Delta^{2}}+\Delta}{N^{2}-1} .
$$

Equation (2) is realized when the energy of $N$ cavity-photons match the $N$-photon transition between the $\mid-/$ and $\mid+/$ levels of the dressed atom ${ }^{29}$, as sketched in Fig. $2 \mathrm{~b}$ for the case $N=$ 2. In the indeterminate case $N=1$, Eq. (2) should be taken in the limit $N \rightarrow 1$, yielding $\omega_{L}=$ $\omega_{a}-\left(2 \Omega^{2}+\Delta^{2} / 2\right) / \Delta$ (in the dispersive regime, $\Delta \neq=0$ ). All the dynamics discussed so far correspond to systems that are Hamiltonian in nature, such as atomic cQED realizations ${ }^{30}$.

\section{Dissipation as a trigger of quantum emission}

Strong dissipation, e.g., in semiconductor CQED, is not always detrimental to quantum effects $^{31,32}$. On the contrary, Purcell enhancement of the Hamiltonian resonances just reported may give rise to giant photon correlations in the statistics of the field detected outside the cavity instead of Rabi oscillations ${ }^{33,34}$. The corresponding zero-delay photon correlations ${ }^{35} g^{(n)}=\left\langle a^{\dagger n} a^{n}\right\rangle /\left\langle a^{\dagger} a\right\rangle^{n}$ are shown in the limit of vanishing pumping in Fig. 3c. An antibunching dip is observed for each $g^{(n)}$ when exciting resonantly the emitter, followed by a series of $N-1$ huge bunching peaks that match the resonances in Eq. (1), plotted in Fig. 3b. In these calculations, the Hamiltonian has been supplemented with superoperators in the Lindblad form to describe dissipation of the cavity (resp. QE) at a rate $\gamma_{a}$ (resp. $\left.\gamma_{\sigma}\right)^{36}$ (see Methods). Parameters used are $\gamma_{a} / g=0.1$ and $\gamma_{d} g=0.01$. Details of the formalism, as well as its extension to describe decoherence, are given in the Supplementary Information. As pumping is increased, resonances in $g^{(n)}$ shift along curves $\omega_{N}(\Omega)$ in the $\left(\omega_{\mathrm{L}}, \Omega\right)$ space defined by Eq. (2). This is shown for $g^{(2)}$ in Fig. 3a for three values of pumping (and animated in the Supplementary Video), starting with $\Omega_{0}=10^{-1} \mathrm{~g}$, close to the vanishing pumping case shown in Fig. $3 \mathrm{c}$. Following $g^{(2)}$ along the $\omega_{2}$ resonance shows that a new peak emerges out of a uniform background, reaching a maximum $g^{(2)} \approx 3649$ at the pumping $\Omega_{1} \approx 4 g$ (middle trace) before a depletion of the resonance forms for higher pumping, reaching its minimum along $\omega_{2}$ of $g^{(2)} \approx 17$ at $\Omega_{2} \approx 32 g$ (background trace). We have thus, so far, transferred some attributes of the remarkable quantum states produced by the $\mathrm{CQED}$ system to the outside world. We now proceed to show in which regimes and in which sense this transfer can actually be used for applications.

\section{Strong correlations do not guarantee useful emission}

The resonances in $g^{(n)}$ are indicative of strong correlations but not in an intuitive way nor in a particularly useful one for applications. Indeed, $g^{(2)}$ (we discuss the case $n=2$ with no loss of generality), is unbounded and cannot be interpreted in terms of probability of two-photon emission. Other quantities to measure correlations, such as the differential correlation function ${ }^{37}$ or the surge ${ }^{38}$, present the same problem. To gain insights into the dissipative context, we turn to a quantum Monte Carlo approach ${ }^{39}$, where one follows individual 
trajectories of the system and records photon clicks whenever the system undergoes a quantum jump. A tiny fraction of such a trajectory is presented in Figs. 4c (a larger fraction is provided in the Supplementary Information). This shows the probabilities of the system to be in the states $\mid n \mathrm{~g} / \mathrm{e} /$ for $n$ up to 2 (probabilities in higher rungs are included in the numerical simulation).

Until time $t \approx 0.8$ (in units of $1 / \gamma_{a}$ ), the QE essentially undergoes fast Rabi flopping (in an empty cavity) under the action of the laser, corresponding to the Mollow regime. At the same time, the driving of the third rung makes the probability to have two photons in the cavity sizable, as seen in bottom panel of Fig. $4 \mathrm{c}$ where the combined probability reaches over $1 \%$, while the probability to have one photon is more than one order of magnitude smaller. This relatively high probability of the two-photon state, given the time available to realize it, eventually results in its occurrence. This causes the emission of a first cavity photon (indicated by a red triangle at the top of the figure) that collapses the wavefunction into the one-photon state, which is now the state with almost unit probability. The system is now expected to emit a second photon within the cavity lifetime (second red triangle in Fig. 4c). There is a jitter in the emission of the two-photon state due to the cavity, but this does not destroy their correlation. After the two-photon emission, the system is left in a vacuum state but without Rabi flopping, that is restored after a direct emission from the QE (black triangle) and a two-photon state is again constructed, preparing for the next emission of a correlated photon pair. The system is then brought back to its starting point. Although one photon coming from the QE decay is emitted per two-photon emission cycle, it is at another frequency and in a different solid angle. The two-photon emission is through the cavity mode, being therefore unspoiled and strongly focused.

Figures $4 \mathrm{~d}-\mathrm{e}$ present a series of detection events such as they would be recorded by a streak camera photodetector ${ }^{40}$ for the pumping values $\Omega_{1}$ and $\Omega_{2}$ of Fig. 3a at $\omega_{2}(\Omega)$. The horizontal axis represents time and each point denotes a detection event as the detection spot is raster scanned across the image. The strong bunching at $\Omega_{1}$ in Fig. 3a conveys that the number of correlated two-photon events (blue points) in Fig. $4 \mathrm{~d}$ is much larger than would be expected for a coherent source. The emission remains nevertheless predominantly in terms of single photons (red points). Whilst the resonances in statistics are strong, they are therefore not meaningful for applications. On the other hand, at $\Omega_{2}$, when the $g^{(2)}$ resonance is depleted, the emission now consists almost exclusively of correlated photon pairs, as can be seen by the dominance of blue points in Fig. 4e.

\section{Definition of a purity of N-photon emission}

Since the standard correlation functions $g^{(n)}$ do not correspond to actual $N$-photon emission, the problem poses itself how to describe what is in fact the most important feature of such an emitter: the amount of $N$-photon emission. Photon-counting ${ }^{41-43}$ is a convenient way to do so in practical terms, since an ideal $N$-photon emitter never produces a number of photons which is not a multiple of $N^{44}$. We observe that for time windows $T$ larger than the coherence time, counting of the photon bundles becomes Poisson distributed, as short time correlations are lost ${ }^{45}$. This distribution is shown in Fig. $4 \mathrm{f}$ for the cases of ideal two-photon (2PE) and three-photon (3PE) emission. However, a non-ideal $N$-photon emitter 
occasionally emits single photons that spoil these distributions. If bundle events are given by the Poisson parameter $\lambda_{N}$ and single events are given by $\lambda_{1}$, one finds (see Methods) that the distribution to count $n$ photons in the time window $T$ is:

$$
P_{N}(n)=e^{-\left(\lambda_{1}+\lambda_{N}\right) T} \sum_{k=0}^{n} \frac{n !\left(\lambda_{1} T\right)^{n-N k}\left(\lambda_{N} T\right)^{k}}{k !(n-N k) !} .
$$

When the suppression of photon emission that is not a multiple of $N$ is efficient, these parameters are related to the cavity population $n_{a}$ through $\lambda_{N}=\gamma_{a} n_{a} / N$. The $\lambda$ parameters being independent of the time window $T$, we can define the purity of $N$-photon emission $\pi_{N}$ as:

$$
\pi_{N}=\lambda_{N} /\left(\lambda_{1}+\lambda_{N}\right)
$$

This ratio represents the percentage of the emission that comes as $N$-photon bundles, that can now be contrasted with $g^{(N)}$, as shown in Fig. $4 \mathrm{~g}$ for $N=2$. Here we find the remarkable result that $g^{(2)}$, often described as the probability for two-photon emission, is in fact anticorrelated with $\pi_{2}$, the actual such probability: when $g^{(2)}$ reaches its maximum, $\pi_{2}$ is starting to grow and when $\pi_{2}$ is maximum, $g^{(2)}$ is locally minimum, although still larger than one.

We characterize the efficiency of $N$-photon emission by plotting the purity and emission together, in Fig. 5a, for $\pi_{2}$ and $\pi_{3}$. Since $N$-photon emission is a $(N+1)$ th order process, it is more easily overcome by dissipation as $N$ increases. However, almost pure two-photon and three-photon emission is already feasible with state of the art cQED systems: $\approx 85 \%$ of twophoton emission can already be obtained with current semiconductor samples $\left(\gamma_{a} / g \approx 0.5, g\right.$ $\approx 12 \mathrm{GHz})^{46,47}$ with a rate over $10^{7}$ counts per second (cps), while circuit QED systems $\left(\gamma_{a} / \mathrm{g} \approx 0.01, g \approx 50 \mathrm{MHz}\right)^{48}$ can even reach $\approx 90 \%$ of three-photon emission with a rate of $10^{3} \mathrm{cps}$. $N$-photon emission takes place when the coupling is large enough for the cavity to stop acting as a mere filter and actually Purcell enhance the corresponding multi-photon transitions ${ }^{49}$. These results are extremely robust against dephasing, thanks to the short time window in which a bundle is generated and emitted, as is shown in the Supplementary Information.

\section{Relation between the bundle and a Fock state}

We have described the emission of our system in terms of "bundles" of photons, introducing a terminology that needs to be justified. In quantum theory, a state of the field with exactly $N$ quanta of excitation is a Fock state $|N\rangle$ and it is natural to question whether our device is not precisely an "Emitter of Fock states $\mid N$ ".

There are subtle links and departures between the two concepts. The Fock state $|N\rangle$ is a welldefined state that can be prepared and maintained exactly. It has no further structure and each of the $N$ photons that compose it is fully indistinguishable from the others. The bundle, on the other hand, arises in a dynamical process of emission, describing the energy released from the cavity QED setup to the outside world. The cavity itself is not in the Fock state $|N\rangle$, being, to begin with, in the vacuum most of the time, and only in very short temporal 
windows does it undergo a cascade that sees in rapid successions the field transit through the various Fock states $|n\rangle$, with $0 \leq n \leq N$, for a time $\gamma_{a} n$ in each of them. Since the system has a small probability to be in the state $|N|$ before the emission and probability close to one to transit through each of the intermediate states during the cascade, one obtains the steady state probability:

$$
p(n)=\frac{n_{a}}{N} \frac{1}{n}, \quad \text { for } \quad 1 \leq n \leq N
$$

A snapshot of the full density matrix in the regime of four-photon emission is given in Fig. 5 b. This shows the breakdown of the matrix into clusters of $2 \times 2$ blocks corresponding to the subspaces of the QE with $n$-photons. The vacuum largely predominates (the probabilities are shown in log-scale), followed by the blocks on the diagonal which provide $p(n)$ as given by Eq. (5), and blocks of coherence between the various manifolds, which are small although nonzero, except the coherence elements $|0 \mu\rangle\langle 4 v$ with $\mu, v \in\{$ g,e $\}$ which are large. This confirms the direct manifestation, also in the dissipative regime, of the quantum superposition of the type $(|0 e\rangle \pm|N e\rangle) / \sqrt{2}$.

There remains a trace of this intra-cavity dynamics in the photodetection. The bundles are strongly correlated in two senses: first extrinsically, the emission comes in groups of $N$ photons, suppressing the release of packets with other numbers of photons. Second, intrinsically, with different time intervals separating successive photons: the first photon is more closely followed by the second one than the second is by the third, and so on till the last photon that comes within $1 / a_{a}$ of the penultimate (see Fig. 5c). Clearly, the bundle is a strongly-correlated group of closely-spaced photons that has a structure which is not described by the abstract object $|N|$ alone. However, regardless of the internal structure of the bundle, it would appear as a Fock state in a measurement integrated over a small time window. Further discussion through the Wigner function can be found in the Supplementary Information.

\section{Regimes of $\mathbf{N}$-photon emission: guns and lasers}

Now that we have engineered $N$-photon emitters, we have to ask the same questions than those put by Glauber ${ }^{35}$ at the dawn of quantum optics, on the nature of quantum optical coherence for these sources. The answer is as simple as it is beautiful: $N$-photon emitters are the exact counterpart of conventional emitters with the provision of replacing the unit of emission - the photon-by a bundle of $N$ of them. We now show that our class of emitters can operate in the same regimes, lasing or photon guns, but with bundles. To do so, we describe the statistics of the bundles when considered as single entities, by introducing the generalized correlation functions $g_{N}^{(n)}$ :

$$
g_{N}^{(n)}\left(t_{1}, \ldots, t_{n}\right)=\frac{\left\langle\mathscr{T}-\left\{\prod_{i=1}^{n} a^{\dagger N}\left(t_{i}\right)\right\} \mathscr{T}+\left\{\prod_{i=1}^{n} a^{N}\left(t_{i}\right)\right\}\right\rangle}{\prod_{i=1}^{n}\left\langle a^{\dagger N} a^{N}\right\rangle\left(t_{i}\right)}
$$


with $\mathscr{T}_{ \pm}$the time ordering operators. This upgrades the concept of the $n$th order correlation function for isolated photons to bundles of $N$ photons. The case $N=1$ recovers the definition of the standard $g^{(n)}$, but for $N \geq 2$, the normalization to the bundle density makes Eq. (6) essentially different from the standard correlation functions $g^{(n \times N)}$. Similarly to the singlephoton case, the two-bundle statistics $g_{N}^{(2)}(\tau)=\frac{\left\langle a^{\dagger N}(0) a^{\dagger N}(\tau) a^{N}(\tau) a^{N}(0)\right\rangle}{\left\langle\left(a^{\dagger N} a^{N}\right)(0)\right\rangle\left\langle\left(a^{\dagger N} a^{N}\right)(\tau)\right\rangle}$ is the most important one. The validity of this definition for $g_{2}^{(2)}$ is confirmed in Figs. $4 \mathrm{~h}-\mathrm{i}$, where it is plotted (smooth curve) along with direct coincidences between clicks from the Monte Carlo simulation (data). Such $g_{2}^{(2)}$ correlations can be measured directly thanks to recent developments in two-photon detection ${ }^{50}$. For the Monte Carlo computation, all events are considered as single photons for the standard $g^{(2)}$ calculation (red curve in Fig. 4h-i), and only two-photon events are considered as the basic unit of emission for $g_{2}^{(2)}$ (blue curve). Except in the small jitter window of width $1 / \gamma_{a}$ in which they cannot be defined, photon pairs exhibit antibunching for long-lived QE, while they are Poisson distributed for short-

lived QE. In the latter case, one can check that $g_{2}^{(3)}\left(\tau_{1}, \tau_{2}\right)=1$ except from the aforementioned jitter window (Supplementary Information). The emitter therefore behaves respectively as a two-photon gun, and-according to Glauber ${ }^{35}$ - as a laser, but at the twophoton level. The effect the QE lifetime has on the statistics of the bundles can be understood as a consequence of the key role the QE emission plays to restore the construction of a $N$-photon state. At the single-photon level, the standard $g^{(2)}(\tau)$ fails to capture this fundamental dynamics of emission. All this confirms the emergence of a new physics at the two-photon level. The same behaviours hold for higher $N$.

\section{METHODS}

\section{System dynamics}

In order to describe dissipation in addition to the Hamiltonian dynamics, we resort to a master equation in the Lindblad form ${ }^{36}$ :

$$
\dot{\rho}=-i[H, \rho]+\left[\frac{\gamma_{a}}{2} \mathscr{L}_{a}+\frac{\gamma_{\sigma}}{2} \mathscr{L}_{\sigma}\right] \rho
$$

expressed in terms of the Liouvillian super-operator $\mathscr{L}_{c} \rho=2 c \rho c^{\dagger}-c^{\dagger} c \rho-\rho c^{\dagger} c$, with $\gamma_{a}$ and $\gamma_{\sigma}$ the decay rates of the cavity and the quantum emitter, respectively. By arranging the elements of the density matrix in a vectorial form, we can express this equation as $\dot{\vec{\rho}}=M \vec{\rho}$ from which the steady-state density matrix $\vec{\rho}_{S S}$ is obtained as the null space of the matrix $M$. A more general expression that takes into account additional dephasing terms is provided and investigated in the Supplementary Information.

For photon counting calculations, we solve the same problem using the Monte Carlo method of quantum trajectories, which allows to use a wavefunction picture in a dissipative context. In this approach, one uses an evolution operator constructed with a non-Hermitian Hamiltonian $H_{e f f}=H-i \frac{\gamma_{a}}{2} a^{\dagger} a-i \frac{\gamma_{\sigma}}{2} \sigma^{\dagger} \sigma$, whose resulting dynamics can be interrupted in 
each time step $\delta t$ by a quantum jump acting on the wavefunction as $c \psi \mid / /\left\langle\psi\left|c^{\dagger} c\right| \psi\right\rangle$ (c $\in\{\mathrm{a}$, $\sigma\})$ with probability $p_{c}=\delta t \gamma c\left\langle\psi\left|c^{\dagger} c\right| \psi\right\rangle$. These jump events are then recorded as photon emissions coming from the cavity or the QE.

\section{Photon counting distribution for the imperfect $\boldsymbol{N}$-photon emitter}

In the limit in which the counting of $N$-photon bundles becomes Poisson distributed, the random variable $X_{N}$ that counts them in a time window $T$ follows the distribution $P\left(X_{N}=k\right)$ $=\exp \left(-\lambda_{N} T\right)\left(\lambda_{N} T\right)^{k / N} /(k / N)$ ! if $k$ is a multiple of $N$, and is zero otherwise, with a generating function $\Pi_{X N}(s)=\left\langle s^{\left.X_{N}\right\rangle}=e^{-\lambda_{N}\left(1-s^{N}\right)}\right.$. A non-ideal $N$-photon emitter emits in addition single photons that spoil these distributions. Photon counting then results from the sum of two random variables $X_{1}+X_{N}$ where $X_{1}$ is a conventional Poisson process. The generating function of the imperfect $N$-photon emitter is $\prod_{X_{1}+X_{N}}=\prod_{X_{1}} \prod_{X_{N}}$, where $\prod_{1}=e^{-\lambda_{1}(1-s)}$ is the generating function of a Poissonian distribution. The closed-form expression provided in the text is straightforwardly derived as $P_{N}(n)=\partial^{(n)} \prod_{X_{1}+X_{N}} /\left.\partial s\right|_{s}=0$.

\section{Supplementary Material}

Refer to Web version on PubMed Central for supplementary material.

\section{Acknowledgments}

We thank J. Sánchez Wol for assistance with Fig. 1. This work was supported by the PolaFlow ERC starting grant, the Marie-Curie project SQUIRREL and the Spanish MINECO (MAT2011-22997). C.S.M. acknowledge a FPI grant, A.G.T and K.M. the Alexander Von Humboldt Foundation and F.P.L. a RyC contract. The authors declare no competing financial interests.

\section{References}

1. Haroche S. Nobel lecture: Controlling photons in a box and exploring the quantum to classical boundary. Rev. Mod. Phys. 2013; 85:1083-1102.

2. Grangier P, Sanders B, Vuckovic. J. Focus on single photons on demand. New J. Phys. 2004; 6

3. Walther H, Varcoe BTH, Englert B-G, Becker T. Cavity quantum electrodynamics. Rep. Prog. Phys. 2006; 69:1325-1382.

4. O’Brien JL, Furusawa A, Vuckovic J. Photonic quantum technologies. Nature Photon. 2009; 3:687695.

5. Afek I, Ambar O, Silberberg Y. High-NOON states by mixing quantum and classical light. Science. 2010; 328:879-881. [PubMed: 20466927]

6. Giovannetti V, Lloyd S, Maccone L. Quantum-enhanced measurements: Beating the standard quantum limit. Science. 2004; 306:1330-1336. [PubMed: 15550661]

7. Denk W, Strickler J, Webb W. Two-photon laser scanning fluorescence microscopy. Science. 1990; 248:73-76. [PubMed: 2321027]

8. Horton N, et al. In vivo three-photon microscopy of subcortical structures of an intact mouse brain. Nature Photon. 2013; 7:205-209.

9. Sim N, Cheng MF, Bessarab D, Jones CM, Krivitsky LA. Measurement of photon statistics with live photoreceptor cells. Phys. Rev. Lett. 2012; 109:113601. [PubMed: 23005626]

10. Ball P. Physics of life: The dawn of quantum biology. Nature. 2011; 474:272-274. [PubMed: 21677723]

11. Brune M, et al. Quantum Rabi oscillation: A direct test of field quantization in a cavity. Phys. Rev. Lett. 1996; 76:1800-1803. [PubMed: 10060524] 
12. Fink JM, et al. Climbing the Jaynes-Cummings ladder and observing its $p_{n}$ nonlinearity in a cavity QED system. Nature. 2008; 454:315-318. [PubMed: 18633413]

13. Kasprzak J, et al. Up on the Jaynes-Cummings ladder of a quantum-dot/microcavity system. Nature Mater. 2010; 9:304-308. [PubMed: 20208523]

14. Jaynes E, Cummings F. Comparison of quantum and semiclassical radiation theory with application to the beam maser. Proc. IEEE. 1963; 51:89-109.

15. Nguyen HS, et al. Ultra-coherent single photon source. Appl. Phys. Lett. 2011; 99:261904.

16. Matthiesen C, Vamivakas AN, Atatüre M. Subnatural linewidth single photons from a quantum dot. Phys. Rev. Lett. 2012; 108:093602. [PubMed: 22463634]

17. Jayakumar H, et al. Deterministic photon pairs and coherent optical control of a single quantum dot. Phys. Rev. Lett. 2013; 110:135505. [PubMed: 23581338]

18. Majumdar A, et al. Linewidth broadening of a quantum dot coupled to an o -resonant cavity. Phys. Rev. B. 2010; 82:045306.

19. Hughes S, Carmichael HJ. Stationary inversion of a two level system coupled to an o -resonant cavity with strong dissipation. Phys. Rev. Lett. 2011; 107:193601. [PubMed: 22181604]

20. Laussy FP, del Valle E, Schrapp M, Laucht A, Finley JJ. Climbing the Jaynes-Cummings ladder by photon counting. J. Nanophoton. 2012; 6:061803.

21. Chough Y-T, Moon H-J, Nha H, An K. Single-atom laser based on multiphoton resonances at far-o resonance in the Jaynes-Cummings ladder. Phys. Rev. A. 2000; 63:013804.

22. Birnbaum K, et al. Photon blockade in an optical cavity with one trapped atom. Nature. 2005; 436:87-90. [PubMed: 16001065]

23. Faraon A, et al. Coherent generation of non-classical light on a chip via photon-induced tunnelling and blockade. Nature Phys. 2008; 4:859-863.

24. Schuster I, et al. Nonlinear spectroscopy of photons bound to one atom. Nature Phys. 2008; 4:382385.

25. Bishop LS, et al. Nonlinear response of the vacuum Rabi resonance. Nature Phys. 2009; 5:105109.

26. Cohen-Tannoudji CN, Reynaud S. Dressed-atom description of resonance fluorescence and absorption spectra of a multi-level atom in an intense laser beam. J. Phys. B. 1977; 10:345-363.

27. Zakrzewski J, Lewenstein M, Mossberg TW. Theory of dressed-state lasers. I. Effective Hamiltonians and stability properties. Phys. Rev. A. 1991; 44:7717-7731. [PubMed: 9905909]

28. Mollow BR. Power spectrum of light scattered by two-level systems. Phys. Rev. 1969; 188:19691975.

29. Gonzalez-Tudela A, Laussy FP, Tejedor C, Hartmann MJ, del Valle E. Two-photon spectra of quantum emitters. New J. Phys. 2013; 15:033036.

30. Gleyzes S, et al. Quantum jumps of light recording the birth and death of a photon in a cavity. Nature. 2007; 446:297-300. [PubMed: 17361178]

31. Volz T, et al. Ultrafast all-optical switching by single photons. Nature Photon. 2012; 6:605-609.

32. Majumdar A, Englund D, Bajcsy M, Vuckovic J. Nonlinear temporal dynamics of a strongly coupled quantum-dot-cavity system. Phys. Rev. A. 2012; 85:033802.

33. del Valle E, Gonzalez-Tudela A, Cancellieri E, Laussy FP, Tejedor C. Generation of a two-photon state from a quantum dot in a microcavity. New J. Phys. 2011; 13:113014.

34. Zubairy MS, Yeh JJ. Photon statistics in multiphoton absorption and emission processes. Phys. Rev. A. 1980; 21:1624-1631.

35. Glauber RJ. The quantum theory of optical coherence. Phys. Rev. 1963; 130:2529-2539.

36. Kavokin, A.; Baumberg, JJ.; Malpuech, G.; Laussy, FP. Microcavities. Oxford Univ. Press; 2011.

37. Kubanek A, et al. Two-photon gateway in one-atom cavity quantum electrodynamics. Phys. Rev. Lett. 2008; 101:203602. [PubMed: 19113340]

38. Hong H-G, Nha H, Lee J-H, An K. Rigorous criterion for characterizing correlated multiphoton emissions. Opt. Express. 2010; 18:7092-7100. [PubMed: 20389730]

39. Plenio MB, Knight PL. The quantum-jump approach to dissipative dynamics in quantum optics. Rev. Mod. Phys. 1998; 70:101-144. 
40. Wiersig J, et al. Direct observation of correlations between individual photon emission events of a microcavity laser. Nature. 2009; 460:245-249. [PubMed: 19587766]

41. Srinivas MD, Davies EB. Photon counting probabilities in quantum optics. Opt. Acta. 1981; 28:981-996.

42. Zoller P, Marte M, Walls DF. Quantum jumps in atomic systems. Phys. Rev. A. 1987; 35:198-207. [PubMed: 9897944]

43. Osad'ko IS. Photon distribution functions of fluorescence photons from single nanoparticles: Three different photon counting methods. Opt. Spectrosc. 2009; 107:1001-1010.

44. Carmichael, H. An open systems approach to Quantum Optics. Springer; 1993. p. 110

45. Loudon, R. The quantum theory of light. Oxford Science Publications; 2000.

46. Ota Y, Iwamoto S, Kumagai N, Arakawa Y. Spontaneous two-photon emission from a single quantum dot. Phys. Rev. Lett. 2011; 107:233602. [PubMed: 22182088]

47. Laucht A, et al. Electrical control of spontaneous emission and strong coupling for a single quantum dot. New J. Phys. 2009; 11:023034.

48. Nissen F, Fink JM, Mlynek JA, Wallra A, Keeling J. Collective suppression of linewidths in circuit QED. Phys. Rev. Lett. 2013; 110:203602. [PubMed: 25167408]

49. del Valle E. Distilling one, two and entangled pairs of photons from a quantum dot with cavity QED effects and spectral filtering. New J. Phys. 2013; 15:025019.

50. Boitier F, Godard A, Rosencher E, Fabre C. Measuring photon bunching at ultrashort timescale by two-photon absorption in semiconductors. Nature Phys. 2009; 5:267-270. 
a

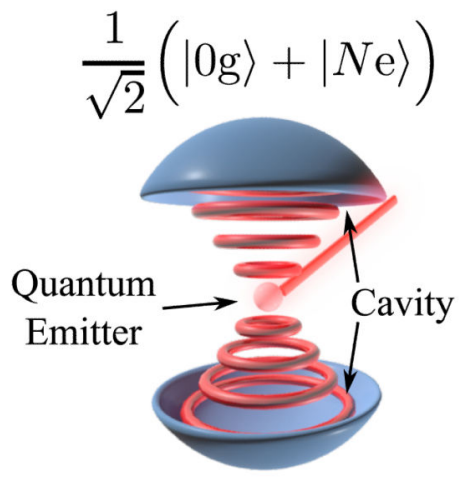

Hamiltonian
cavity QED

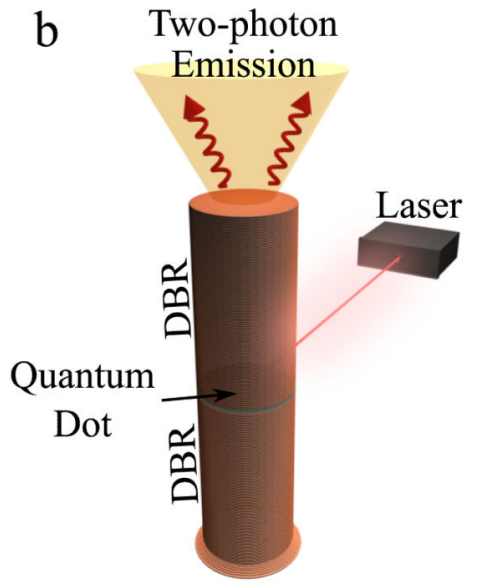

\section{Dissipative microcavity QED}

FIG. 1.

a, cQED allows to bring Quantum Electrodynamics-the theory of light-matter interaction - under prolonged scrutiny at the level of a few photons and in the presence of a quantum emitter. An external laser can be shined on the emitter to drive its dynamics. We show in this text how peculiar quantum superpositions can thus be realized and the emission subsequently forced to take place exclusively in bundles of $N$-photons. b, A possible solid state implementation of our proposal places a quantum dot in a micropillar: exciting on the side with a conventional laser, one can collect in the cavity emission the output from a quantum laser or a quantum gun, depending on the system parameters. 
a

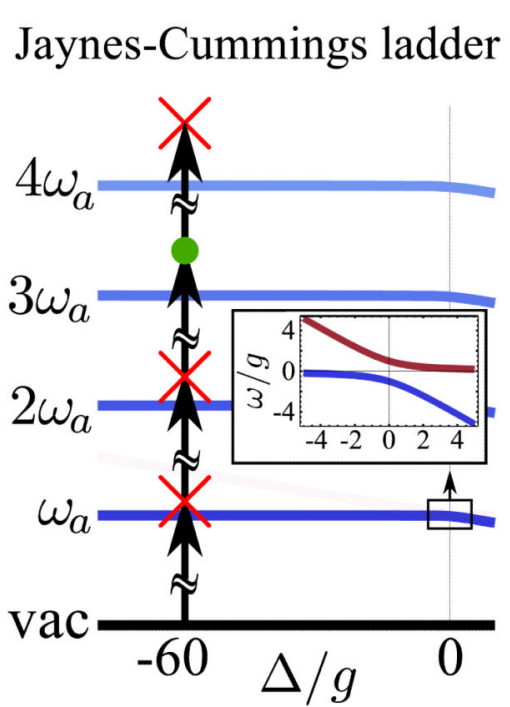

b

Mollow ladder

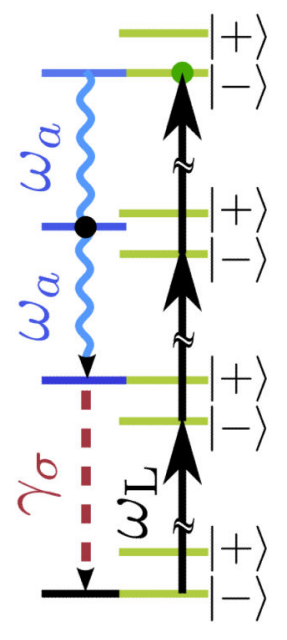

FIG. 2.

Energy levels of the two limiting cases of excitation: a, In the low excitation regime, the Jaynes-Cummings ladder (anticrossing magnified in inset) is probed by resonantly exciting a given rung of the ladder, with photon blockade at all others. b. In the high excitation regime: the laser dresses the QE while the cavity Purcell-enhances a $N$-photon transition from $\mid->$ to $|+\rangle$ (here for $N=2$ ). A subsequent emission from the $\mathrm{QE}$ brings the system back to a $\mid->$ state. 


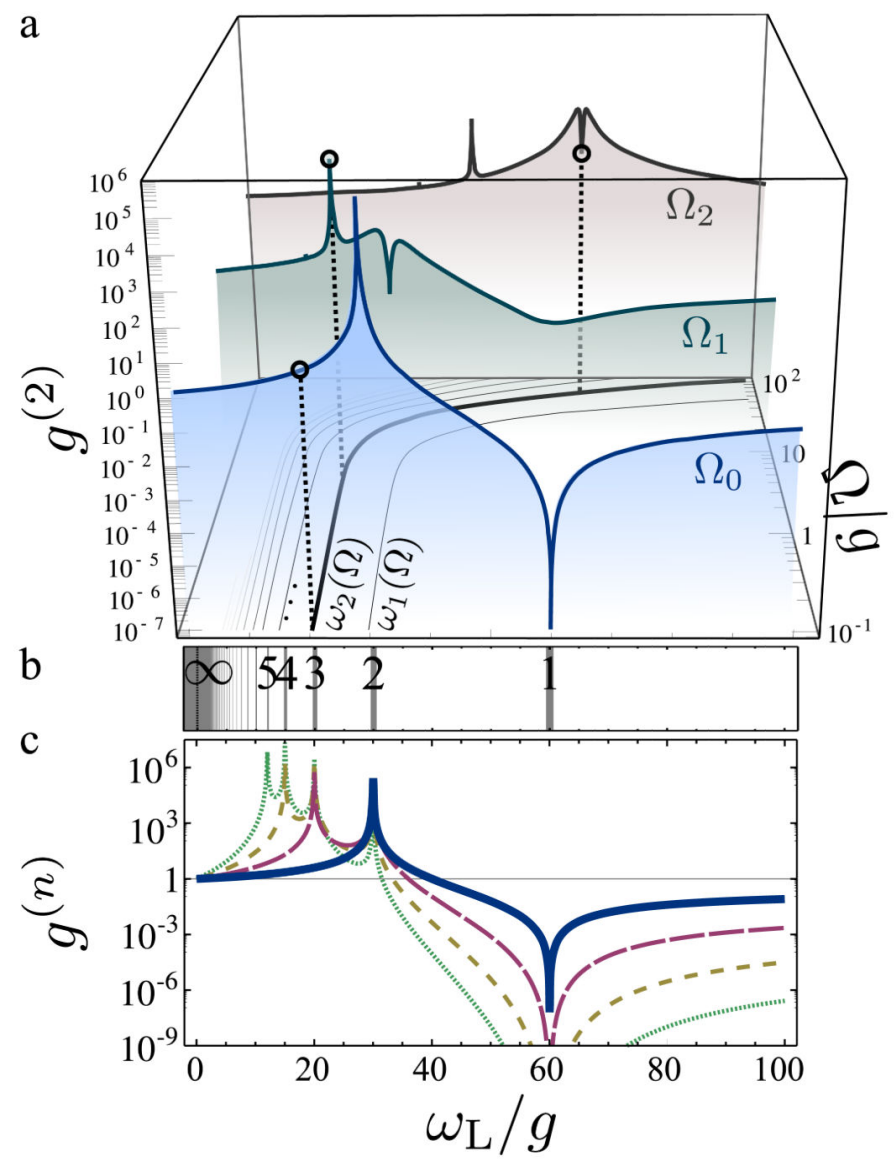

FIG. 3.

a, $g^{(2)}$ as a function of $\omega_{\mathrm{L}}$ for pumping $\Omega_{0} \approx 10^{-2} g, \Omega_{1} \approx 4 g$ and $\Omega_{2} \approx 32 g$. The resonances $\omega_{N}(\Omega)$ are shown in the plane $\left(\omega_{\mathrm{L}}, \Omega\right)$. Open circles are the projection of $\omega_{2}$ on $g^{(2)}$. $\mathbf{b}$,

Resonant energies to excite the $n$th rung of the ladder. c, $g^{(n)}$ for $n=2$ (solid), 3 (long dash), 4 (short dash) and 5 (dotted) at vanishing pumping with $n-1$ bunching resonances matching those in b. $\Delta g=-60$ in all the panels. 


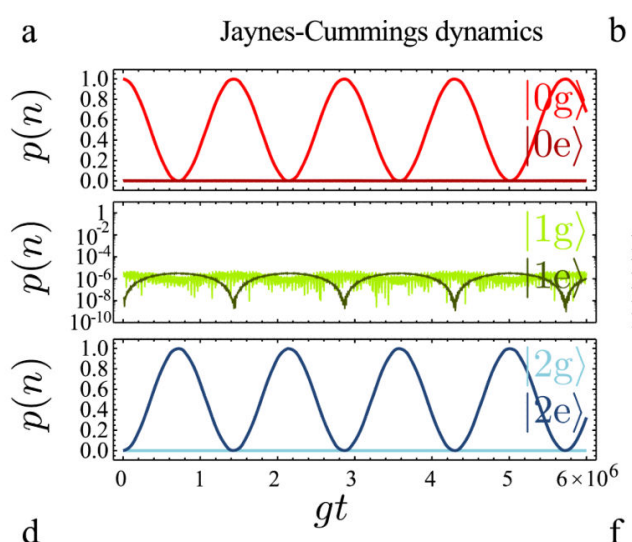

d
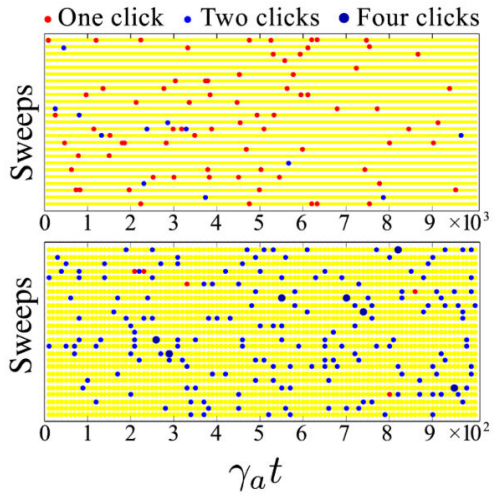
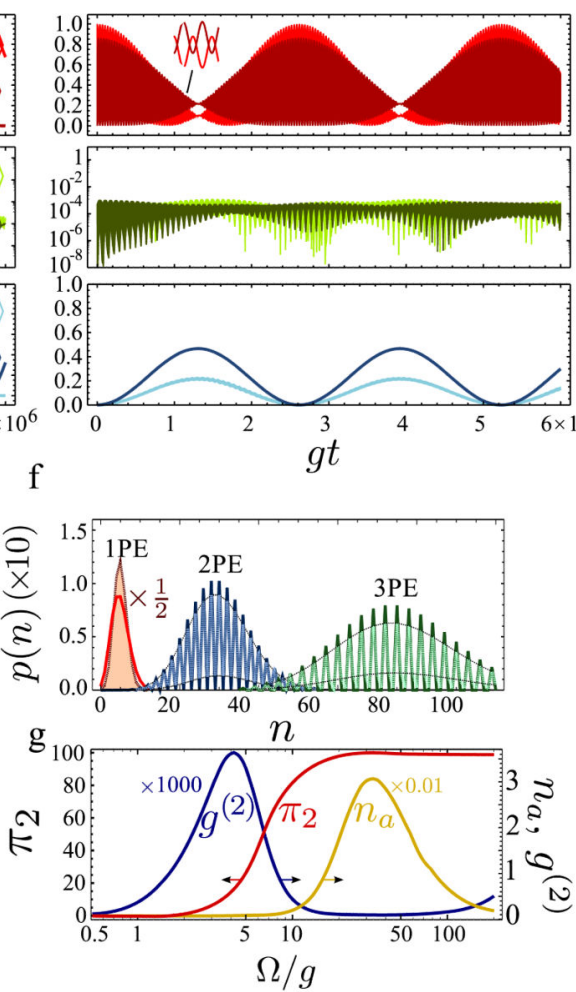

C
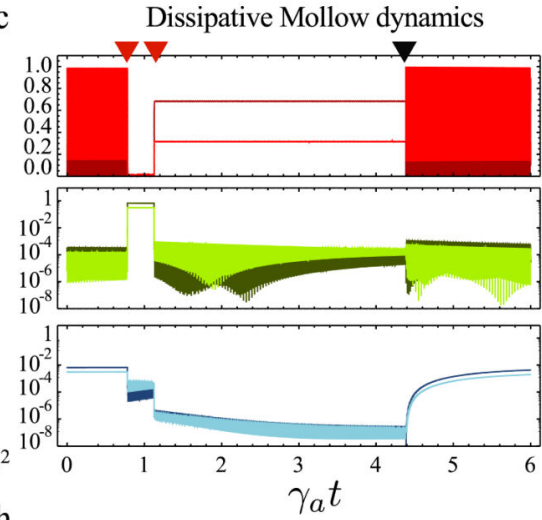

$\mathrm{h}$
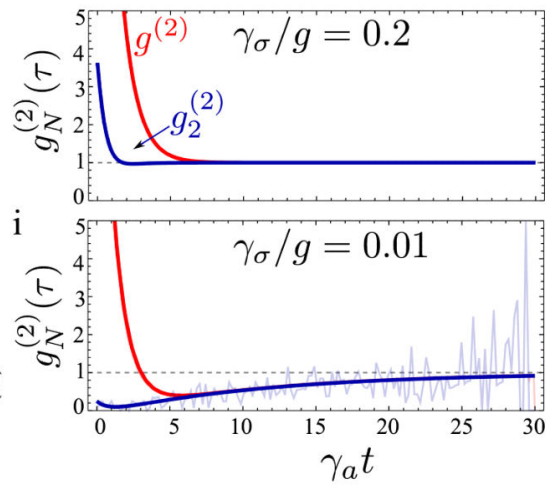

FIG. 4.

Upper row: Wavefunction evolution at the two-photon resonance pictured through the probability of the system to be in any of the states $\mid n \mathrm{~g} / \mathrm{e} /$. a, Hamiltonian evolution in the Jaynes-Cummings regime (low pumping). b. Hamiltonian evolution in the Mollow regime (high pumping). c, Quantum trajectory during a two-photon emission in the same regime as in $\mathrm{b}$, but in the presence of dissipation. $\mathbf{d}-\mathbf{e}$, Cavity-photon clicks as they would be recorded by a streak camera ( 25 sweeps shown) for the pumping values $\Omega_{1}$ in d and $\Omega_{2}$ in e at $\omega_{2}$. In d the emission is highly bunched although it largely consists of single clicks, $g^{(2)}=3649$ and $\pi_{2}=16 \%$, while in e, $g^{(2)}=17$ with $\pi_{2}=98.8 \%$. f, Ideal $N$ PE ( $N$-Photon Emission $)$ in thick lines and $99 \%$ NPE in translucid lines with an envelope to guide the eye. g, Pumping dependence of, left axis, $\pi_{2}$ and, right axis, $g^{(2)}$ (from 0 to 3 649) and $n_{a}$ (from 0 to 0.03 ) following $\omega_{2} . \mathbf{h}-\mathbf{i}$, Second order photon correlations at the $N=1$ (red) and $N=2$ (blue) level, from Eq. (6) (smooth curve) and from Monte Carlo clicks (data). 
a

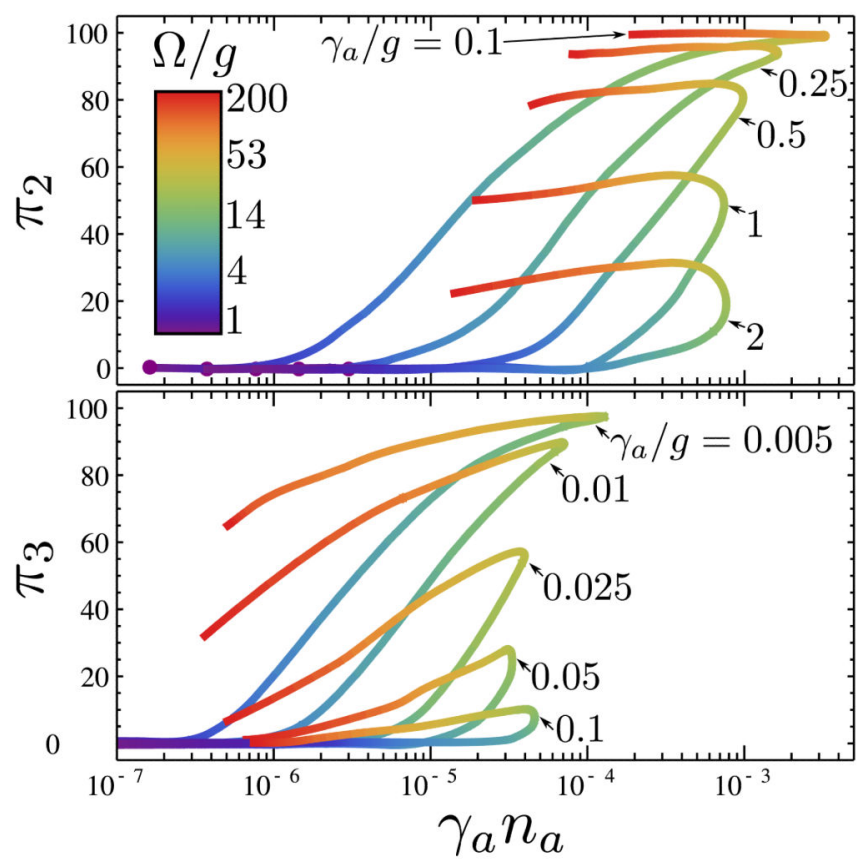

$\mathrm{b}$

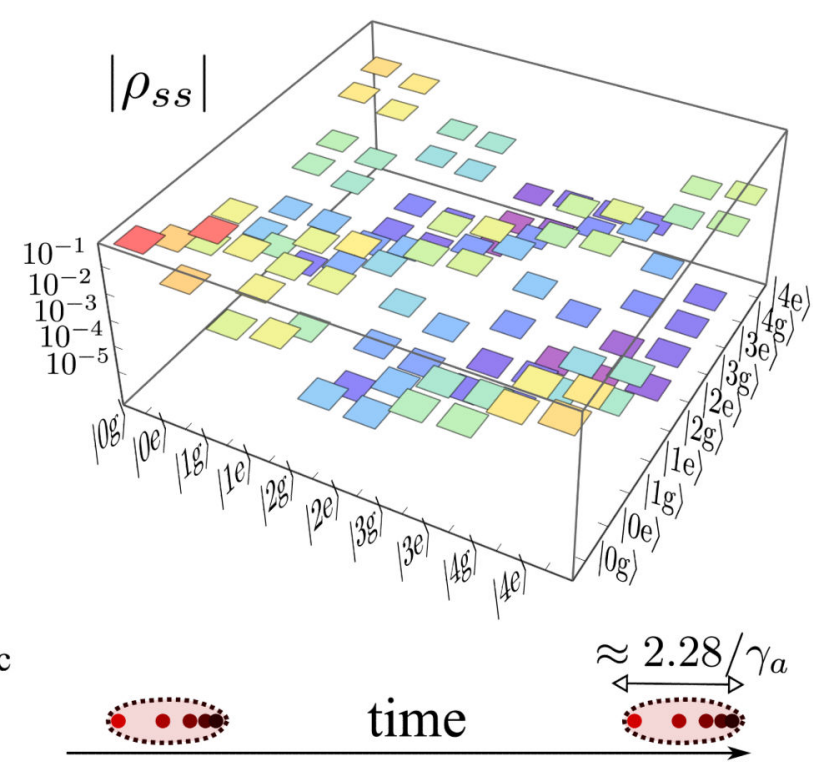

FIG. 5.

a, Figures of merit for two- and three-photon emission in the space of purity/emission intensity. Almost pure two-photon and three-photon emission can be achieved with state of the art cQED samples: $\gamma_{d} g=0.01$ for $\pi_{2}$ and 0.001 for $\pi_{3}$. b, Full density matrix of the system in the regime of four-photon emission, showing the predominance of the vacuum and the strong coherence between the $2 \times 2$ sub-blocks of 0 and 4 photons, and the $1 / n$ cascade along the diagonal. c, Sketch of two five-photon bundles. Each bundle is composed of photons that pile up together upfront in time, due to the mechanism of their production. This structure is not described by the state $|5\rangle$. 